ARTICLE

https://doi.org/10.1038/s41467-019-13738-6

\title{
Two-dimensional nanoframes with dual rims
}

\author{
Sungjae Yoo ${ }^{1}$, Jeongwon Kim ${ }^{1}$, Sungwoo Choi $^{2}$, Doojae Park $^{2} \&$ Sungho Park ${ }^{1 \star}$
}

The synthesis of highly complex two-dimensional (2D) metal nanoframes remains a great challenge. Synthetic strategies for preparing 2D metal nanoframes are few, and rational and systematic synthetic pathways to more complicated architectures have not yet been reported. Herein, we demonstrate a stepwise synthetic strategy for complex 2D metal nanoframes with a high degree of intricacy; the strategy leads to a variety of shapes, including rings, triangles, hexagons, and tripods with tailorable single or double frames in a single entity. These nanoframes of high homogeneity could be obtained through selective combination of four different chemical toolkits consisting of selective etching and deposition on certain facets, and concentric and/or eccentric regrowth by controlling the mismatches of lattice constants of metals. The resulting nanoframes were highly homogeneous in size and shape and had van der Waals interactions that maximized rim-to-rim contact, allowing them to uniquely self-assemble into large-area superstructures.

\footnotetext{
${ }^{1}$ Department of Chemistry, Sungkyunkwan University, Suwon 440-746, South Korea. ${ }^{2}$ Department of Applied Optics and Physics, Hallym University, Chuncheon 24252, South Korea. *email: spark72@skku.edu
} 
S imilar to the fact that all planets are spherical, owing to the gravity well centered on the body's center of mass, in most cases the shapes of metallic nanoparticles are likewise limited to spherical geometry owing to self-stabilization that minimizes their exposed surface area. The synthesis of anisotropic nanoparticles that deviate from this spherical shape, and especially nanoplate-like architectures ${ }^{1-5}$, remains a great challenge. Further, producing nanoplates with structural diversity and complexity in a rational and systematic fashion is very rare. To synthesize sophisticated architectures of high-order complexity and homogeneity ${ }^{6-10}$, tour de force synthetic methods are required. Multiple-step reactions are the typical strategy used to synthesize such complex nanostructures, although the examples referenced above often adopt repeated instances of the same synthetic steps, resulting in limited success in forming complex nanoparticles. Sequential reactions composed of multiple distinct synthetic pathways ${ }^{11}$ and the ability to combine such steps on demand will offer a powerful tool for further expanding the variety of $2 \mathrm{D}$ nanoplate architectures with a high degree of intricate features, mirroring the synthetic strategies often adopted in the total synthesis of complex organic molecules. Herein, we demonstrate a rational and stepwise synthetic strategy for nanoplates with an high degree of intricacy, leading to a gallery of various shapes such as rings, triangles, hexagons, and tripods, and having tailorable single or double frames. These high homogeneous nanoframes can be obtained through selective combinations of four different chemical toolkits consisting of selective etching, rim-on deposition, and concentric and/or eccentric regrowth.

\section{Result and discussion}

Synthetic strategy of 2D complex nanoframes. In the selective etching step, one component could be etched out leaving the other component almost intact, which is useful for obtaining frameworks. In addition, the selective etching could be performed on the reshaping process of the synthesized nanoparticles. For instance, the tips or protruded domains of nanoparticles could be selectively etched out, leading to the rounded-shaped of nanoparticles, which opens up the way of reshaping the nanoparticles. In the step of rim-on deposition, one can control the way of deposition of another component on the certain facets of template nanoparticles taking advantage of surface energy and/or coordination number difference. Typically, $\mathrm{Pt}^{4+}$ ions are more likely reduced on the facets with the lower coordination number like the edge and vertex, as compared to the terrace of $\mathrm{Au}$ nanoparticles. Another important synthetic strategy is the control of regrowth direction. In our $2 \mathrm{D}$ nanoframes, the complexity of nanoframes could be further evolved by controlling the regrowth pattern of $\mathrm{Au}$ around $\mathrm{Pt}$ skeleton. When $\mathrm{AuCl}_{4}{ }^{-}$ions are added to reaction solution containing the two-component PtAu nanoparticles in the presence of reductants, the $\mathrm{AuCl}_{4}{ }^{-}$ions are selectively reduced on Au domains, due to the large lattice constant mismatch between $\mathrm{Au}$ and $\mathrm{Pt}$ (vide infra). However, the homogeneous deposition of $\mathrm{Au}$ on PtAu nanoparticles is also feasible by depositing a thin layer of $\mathrm{Ag}$ (as a mediator reducing the lattice mismatch between $\mathrm{Pt}$ and $\mathrm{Au}$ ) on Pt. By adopting this synthetic strategy, one can control the Au regrowth direction around PtAu nanoframes, saying eccentric or concentric growth (vide infra). Importantly, one can combine these four synthetic steps and perform them multiple times to control the complexity of nanoparticles as following; (1) Selective-etching: one metal is selectively etched to ions, leaving the other metal almost intact. (2) Rim-on-deposition: metal ions are exclusively reduced at the periphery of the core plate. (3) Concentric-growth: metal atoms are homogeneously deposited around the metal frames. (4)
Eccentric-growth: metal atoms are selectively deposited to certain domains of metal frames wherein the difference of lattice constants of metals is absent.

Fine-control of 2D Au nanoplate morphology. Figure 1 shows the complete chemical synthetic pathways for complex 2D metal nanoframes, starting from triangular Au nanoplates and going through multiple synthetic steps on demand, systematically leading to complex 2D nanostructures with double nanoframes. $\mathrm{Au}$ nanoplates of various shapes (triangle, disk, hexagon, and tripod), as represented in the scheme, were adopted as starting templates en route to $2 \mathrm{D}$ complex nanoframes. Fine-tuning in the resulting shapes could be achieved by controlling the etching and growth parameters precisely (vide infra). We designate the corresponding shape-adjustment steps as selective etching, selective growth, and overgrowth modes, as described in the central region of Fig. 1. In the selective etching step, $\mathrm{AuCl}_{4}{ }^{-}$ions were added to the triangular nanoplates solution; the $\mathrm{Au}$ nanoplates initially showed sharp three vertices and gradually became truncated $\left(\mathrm{AuCl}_{4}{ }^{-}+2 \mathrm{Au}+2 \mathrm{Cl}^{-} \rightarrow 3 \mathrm{AuCl}_{2}^{-}\right)$, eventually leading to disks. It also implies that the standard redox potential of the oxidation of vertex is lower than that of $\mathrm{AuCl}_{4}{ }^{-}$and therefore the oxidation of vertex $\left(\mathrm{Au}_{\text {vertex }}(\mathrm{s})+\mathrm{s} 2 \mathrm{Cl}^{-} \rightarrow \mathrm{AuCl}_{2}{ }^{-} \mathrm{uC} 2 \mathrm{e}^{-}\right)$occurs as $\mathrm{AuCl}_{4}{ }^{-}$ ions become reduced ${ }^{12}$. In the overgrowth step, when the chemical environment was changed to mild reduction conditions by slowly adding ascorbic acid, Au atoms preferred to be deposited on the edges of disks (which had higher surface energy compared to flat terraces) and the shape evolved to a hexagonal shape. Alternatively, in the selective growth step, a thin layer of Ag was formed on the $\{111\}$ facets (top and bottom terraces) of triangular $\mathrm{Au}$ nanoplates in the presence of $\mathrm{I}^{-}$ions, and then ascorbic acid (reducing agent) and $\mathrm{AuCl}_{4}^{-}$ions were added sequentially. It is noteworthy that $\mathrm{AuCl}_{4}{ }^{-}$ions are reduced to $\mathrm{AuCl}_{2}{ }^{-}$ions in the presence of ascorbic acid. The existence of a thin Ag layer on $\mathrm{Au}$ nanoplates determined the growth mode of $\mathrm{Au}$, exclusively on the tips, preventing the $\mathrm{Au}$ growth on terraces., whereby $\mathrm{Ag}^{+}$ions were reduced again to a thin Ag layer by ascorbic acid. At the same time, the edge sites of $\mathrm{Au}$ nanoplates were oxidized by residual $\mathrm{AuCl}_{4}{ }^{-}$ions (still unreacted with ascorbic acid), leading to partial etching of edges, and thus eventually to tripods ${ }^{13,14}$. It is noteworthy that the observed shape transformation of triangular $\mathrm{Au}$ nanoplates proceeded without any new nucleation events in all synthetic processes. The average size of the newly formed nanoplates depends on the amount of etchants $\left(\mathrm{AuCl}_{4}{ }^{-}\right.$for disks) and/or precursors $\left(\mathrm{AuCl}_{2}{ }^{-}\right.$for hexagons and tripods) present in the solution. Typically, the diameter (distance from the center to the tip) can be tuned within the range from $78 \pm 5 \mathrm{~nm}$ to $54 \pm$ $5 \mathrm{~nm}$ and the thickness is from $18 \pm 1 \mathrm{~nm}$ to $10 \pm 2 \mathrm{~nm}$ (Supplementary Fig. 1).

Synthetic steps in the synthesis of 2D complex nanoframes. In Fig. 1, each distinctive step in the synthesis of complex nanoframes is described by arrows having different colors for easier recognition; these are listed as follows. (1) Rim-on deposition (black arrow): metal ions are exclusively reduced at the periphery of the core plate. (2) Selective etching (green arrow): one metal is selectively etched to ions, leaving the other metal almost intact. (3) Eccentric growth (orange arrow): metal atoms are selectively deposited to certain domains of metal frames where there is no difference in the lattice constants of metals. (4) Concentric growth (purple arrow): metal atoms are homogeneously deposited around the metal frames. By following the schematic pathways described in Fig. 1, we will describe how one can synthesize the complex $2 \mathrm{D}$ nanoframes with dual rims in a single entity, composed of six steps as follows. 


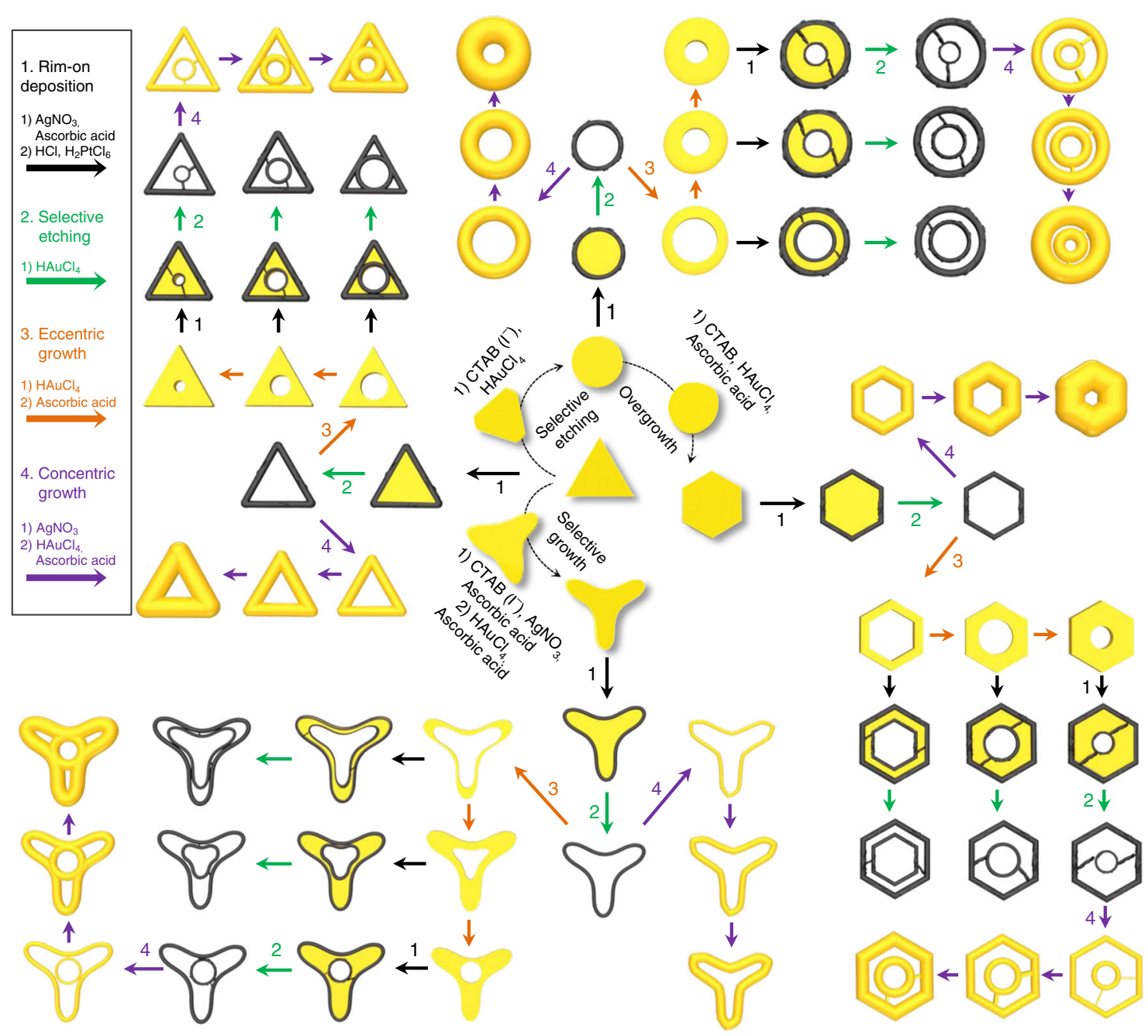

Fig. 1 Schematic illustration of the synthetic pathways of complex 2D nanostructures. The multistep reactions to synthesize complex 2D metal nanoframes involved rim-on deposition of Pt (black arrow), selective etching (green arrow), eccentric growth (orange arrow), and concentric growth (purple arrow).

A section for rim-on deposition. In step one (rim-on deposition), when $\mathrm{Pt}^{4+}$ ions were added to Au nanoplates coated with thin layer of Ag (triangle, disk, hexagon, or tripod shapes) in the presence of ascorbic acid, the galvanic replacement reaction between $\mathrm{Pt}^{4+}$ ions and the thin layer of $\mathrm{Ag}$ on $\mathrm{Au}$ surface occurred exclusively on the vertices and edges due to the higher surface energy of $\{112\}$ facets (i.e., the vertices and edge) compared to $\{111\}$ facets (i.e., the terraces on top and bottom) of nanoplates (Supplementary Fig. 2) ${ }^{15}$. The thickness of Pt rims at the peripheries could be tailored by the total amount of $\mathrm{Pt}^{4+}$ ions added to the reaction solution. Typically, we controlled the Pt rim thickness to be around $11 \pm 2 \mathrm{~nm}$ (disk), $12 \pm 3 \mathrm{~nm}$ (triangle), $11 \pm 2 \mathrm{~nm}$ (hexagon), and $10 \pm 4 \mathrm{~nm}$ (tripod) (Supplementary Fig. 3).

A section for selective etching. Next, in step two (selective etching), the central bulk Au domains were selectively etched to $\mathrm{Au}^{+}$by means of adding $\mathrm{AuCl}_{4}{ }^{-}$ions $\left(\mathrm{AuCl}_{4}{ }^{-}+2 \mathrm{Au}^{0}+2 \mathrm{Cl}^{-} \leftrightarrow\right.$ $3 \mathrm{AuCl}_{2}^{-}$), while the $\mathrm{Au}$ adatoms in close proximity to Pt rims remained intact, leaving a PtAu framework (Fig. 2a, e, i, and m). The incomplete etching of inner Au domains to a very thin layer was readily achievable and controllable, possibly because the $\mathrm{Au}$ adatoms at the boundary between the $\mathrm{Pt}$ rim and $\mathrm{Au}$ domain intercalate to form an alloy between $\mathrm{Pt}$ and $\mathrm{Au}$ at the boundary, leaving a very thin layer of $\mathrm{Au}$ in the inner rims. Energydispersive spectrometry (EDS) mapping images revealed that there was ca. $73.95 \%$ and $26.05 \%$ (disk), $52.61 \%$ and $47.39 \%$ (triangle), $58.96 \%$ and $41.04 \%$ (hexagon), and $45.96 \%$ and $55.04 \%$ (tripod) Pt and Au atoms, respectively, in the single nanoframes (Supplementary Fig. 3). As in the case of single nanoframes, the complete removal of $\mathrm{Au}$ was not feasible due to $\mathrm{PtAu}$ alloy formation. Although elemental mapping showed $\mathrm{Au}$ on the Pt rims, the pure $\mathrm{Au}$ was too thin to show the corresponding optical characteristics of $\mathrm{Au}$, as evident from the surface plasmon profile of the single nanoframes acquired by means of UV/Vis-NIR spectroscopy (Supplementary Fig. 4). When the Au atoms were deposited on the rims of $2 \mathrm{D} \mathrm{PtAu}$ single nanoframes, there were two alternative growth patterns (i.e., concentric or eccentric growth modes). In the eccentric growth mode, $\mathrm{Au}$ atoms were preferentially deposited on the inner regions of the PtAu nanoframes. In contrast, in the concentric growth mode, $\mathrm{Au}$ atoms were deposited almost equally on all directions. These two different growth patterns could be directed by controlling the reaction parameter (vide infra).

A section for eccentric growth. In step three (eccentric growth), when $\mathrm{AuCl}_{4}{ }^{-}$ions were added to PtAu nanoframes, the residue of $\mathrm{Au}$ on the inner rims acted as a nucleus and the $\mathrm{AuCl}_{4}{ }^{-}$ions were 

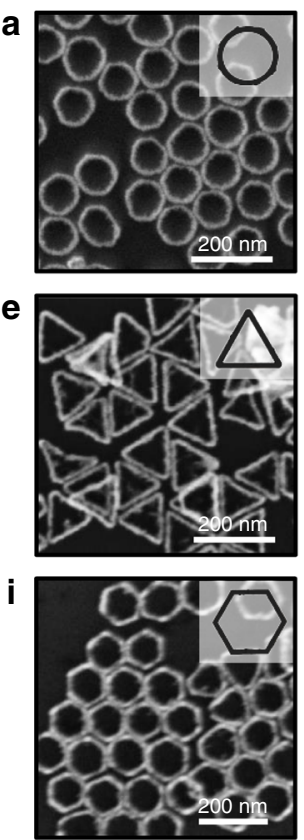

m

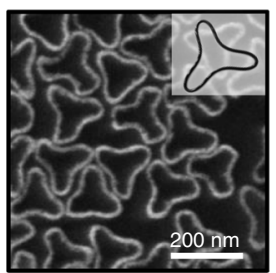

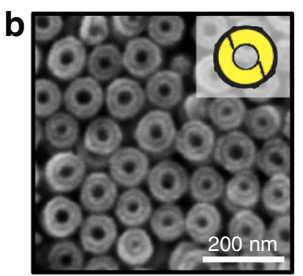
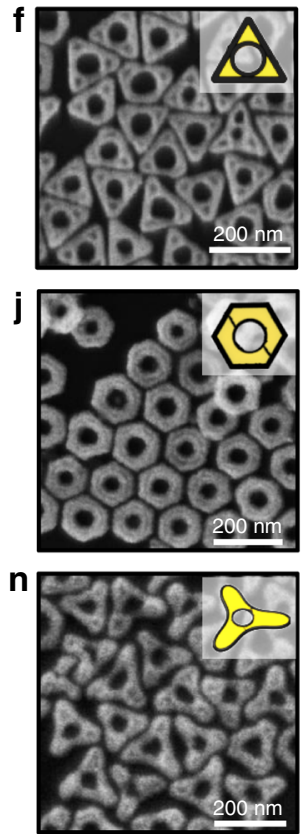
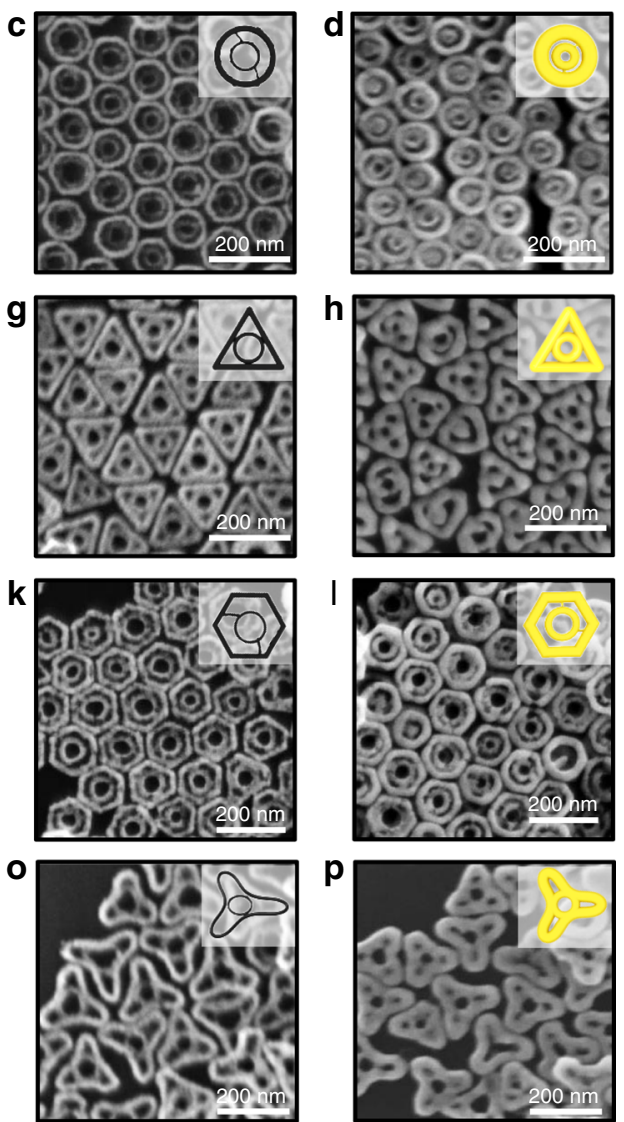
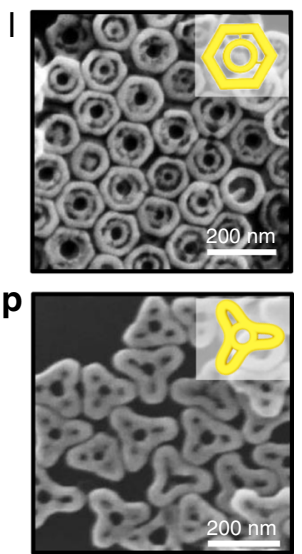

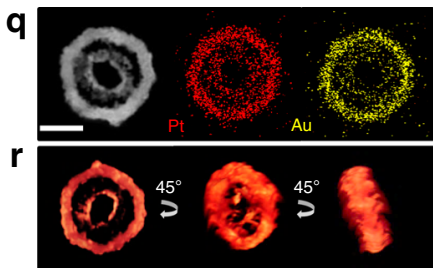

$\mathbf{S}$

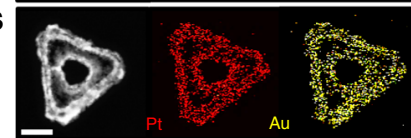

$t$
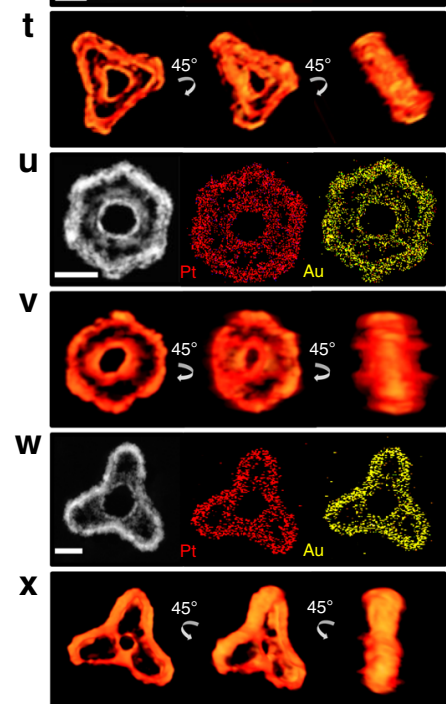

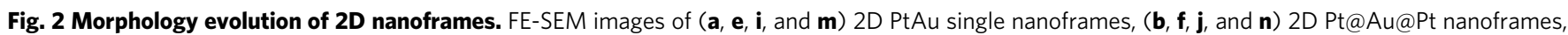
(c, $\mathbf{g}, \mathbf{k}$, and $\mathbf{o}$ ) 2D PtAu double nanoframes, and (d, h, I, and $\mathbf{p}$ ) 2D Pt@Au double nanoframes with different shapes. (q, s, u, and w) TEM images and EDS image mappings of 2D PtAu double nanoframe with different shapes (scale bars: $50 \mathrm{~nm}$ ). (r, $\mathbf{t}, \mathbf{v}$, and $\mathbf{x}$ ) 3D visualizations of 2D PtAu double nanoframes from the STEM tomography.

selectively reduced on $\mathrm{Au}$ domains, resulting in homogeneous inward Au growth (Fig. 3b-e). As Au growth proceeded, there was no noticeable change in the outer diameter (from $97 \pm 6 \mathrm{~nm}$ to $100 \pm 7 \mathrm{~nm}$ ); rather, the inner diameter of the empty holes decreased while retaining its circular shape, indicating that the growth mode followed the Frank-van der Merwe mode, wherein adatoms attach preferentially to surface sites to produce a smooth and fully formed layer (Supplementary Fig. 5). It is noteworthy that the growth of Au in the outward direction was not favorable because the lattice constant mismatch between $\mathrm{Au}$ and $\mathrm{Pt}$ is relatively large (i.e., the lattice constant of $\mathrm{Au}$ and $\mathrm{Pt}$ is $0.4065 \mathrm{~nm}$ and $0.3912 \mathrm{~nm}$, respectively). EDS line mapping (Fig. 3, below each TEM image) showed that, regardless of shape, the $\mathrm{Pt}$ domains were dominantly located on the outer sides of rims, whereas $\mathrm{Au}$ resided on the inner sides of rims. Importantly, the inner $\mathrm{Au}$ domain thickness could be controlled precisely by controlling the total amount of $\mathrm{Au}^{3+}$ ions, allowing one to control the diameter of inner empty holes. Typically, for rings, the thickness of Au domains was controlled from $19 \pm 3 \mathrm{~nm}$ to $39 \pm$ $3 \mathrm{~nm}$ (Supplementary Fig. 5).

In step four (rim-on deposition), again, when $\mathrm{Pt}^{4+}$ ions were added to $\mathrm{Au}$ rings (triangle, disk, hexagon, or tripod shapes) in the presence of ascorbic acid, Pt atoms were deposited on both the inner and outer peripheries, excluding deposition on the flat tops and bottoms of Au domains. The resulting Pt@Au@Pt single nanoframes are shown in Fig. 2b, f, j, and n(second column).

In step five (selective etching), the inner Au domains were selectively etched to $\mathrm{Au}^{+}$by the addition of $\mathrm{AuCl}_{4}{ }^{-}$ions, leaving a
PtAu framework with double rims in one entity, as shown in Fig. 2c, g, k, and o (third column). It is remarkable that the inner rims were interconnected with the outer rims through the scattered PtAu islands without the loss of integrity of the double frame architecture (for ring and hexagon shapes). For triangles and tripods, triangle-inscribed and tripod-inscribed circles were clearly observed to be in contact with the three corresponding edges. The intricacy of double frames could be evolved further by means of controlling the thickness of inner $\mathrm{Au}$ domains as described in Fig. 3j ${ }^{16-19}$ Strikingly, the inner rim-to-outer rim distance could be tailored by controlling the widths of the inner Au domains. Typically, the rim-to-rim distance for a disk shape was tunable from $8 \pm 2 \mathrm{~nm}$ to $19 \pm 4 \mathrm{~nm}$ (Fig. $3 \mathrm{k}-\mathrm{m}$ ). For the triangle shape, the three holes at the tips were distinctive when the tip-to-inner rim distance was $17 \pm 5 \mathrm{~nm}$, and they gradually merged as the tip-to-inner rim distance approached $26 \pm 5 \mathrm{~nm}$ (Fig. $3 n-p)$. This tunability was applicable to hexagons and tripods as well (Fig. 3q-v). Low-magnification field-emission scanning electron microscopy (FESEM) images revealed that the as-prepared double nanoframes were extraordinarily homogeneous in both shape and size, indicating the efficacy and controllability of the suggested multistep synthetic routes (Supplementary Fig. 6). The morphologies of PtAu double nanoframes were further analyzed in detail from different angles using angle-dependent scanning transmission electron microscopy (STEM) tomography (Fig. 2r, t, v, x and Supplementary Movies 1-4), and their two-dimensional geometry and compositional information were determined (Supplementary Fig. 7). 


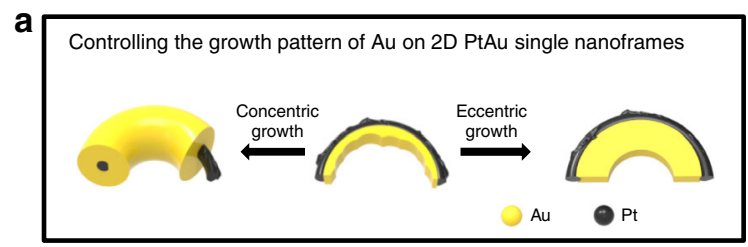

b
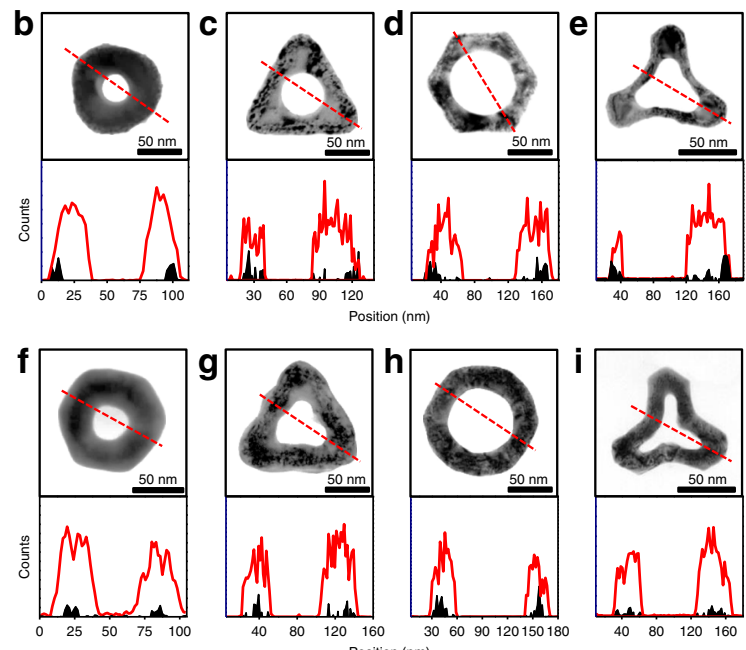

Position (nm)
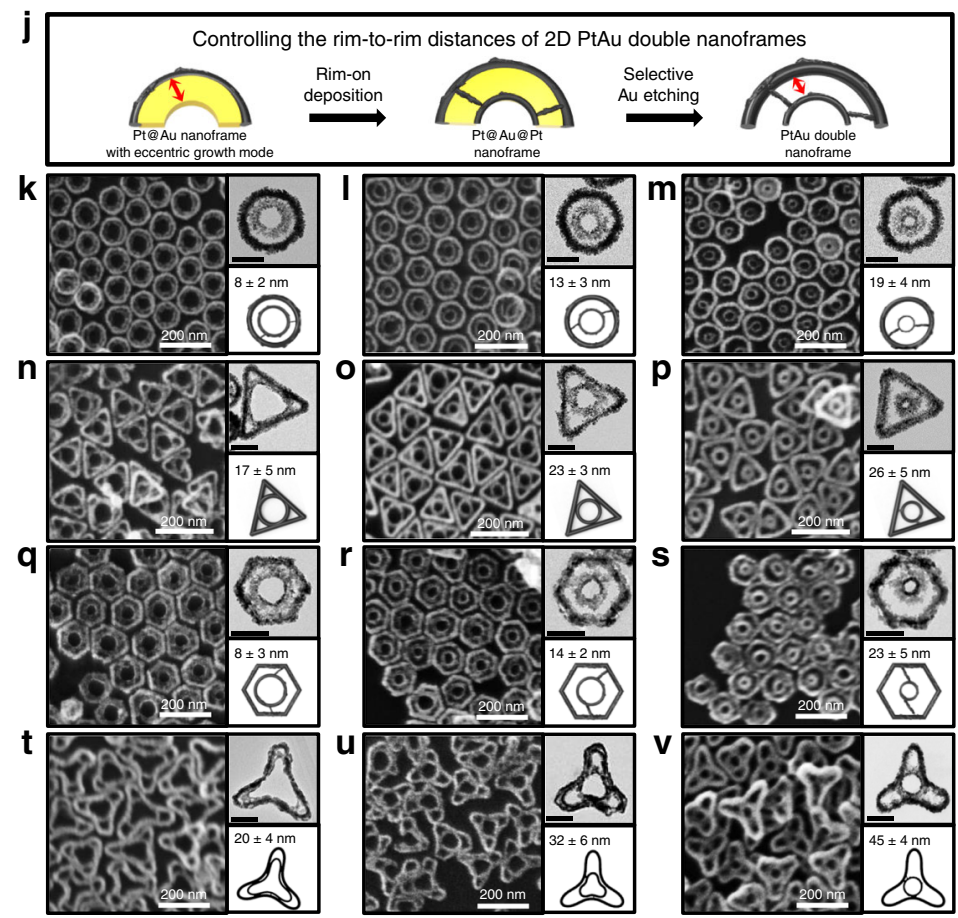

$\mathrm{p}$
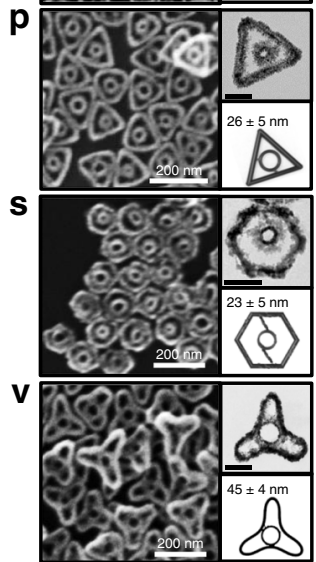

Fig. 3 Controlling the growth pattern of Au and rim-to-rim distances of PtAu double nanoframes. a Schematic illustration shows the mechanism of the growth pattern control of Au on 2D PtAu single nanoframes. TEM images and line mapping profiles show 2D Pt@Au nanoframes grown in (b-e) eccentric and $(\mathbf{f}-\mathbf{i})$ concentric growth modes. Line mapping was conducted along the red lines in TEM images. $\mathbf{j}$ Schematic illustration of shows the synthesis procedure of 2D PtAu double nanoframes with various shape and rim-to-rim distances. k-v FE-SEM images and TEM images show 2D PtAu double nanoframes having various rim-to-rim distances in each shape. Morphology and dimension information (rim to rim distances) are given below each TEM image (scale bars: $50 \mathrm{~nm}$ ).

A section for concentric growth. In step six (concentric growth), when $\mathrm{AuCl}_{4}{ }^{-}$ions were added to Pt nanoframes in the presence of ascorbic acid, the $\mathrm{AuCl}_{4}^{-}$ions were homogeneously deposited around the $\mathrm{Pt}$ rims, resulting in $\mathrm{Au}$ growth both inward and outward. The Ag present on the Pt rims played the role of a mediator, reducing the difference in lattice constants between $\mathrm{Pt}$ and $\mathrm{Au}$ and thereby allowing the deposition of Au not only on $\mathrm{Au}$ but also on Pt to an equal extent (note that the lattice constant of $\mathrm{Ag}$ is $0.4079 \mathrm{~nm}$ ). We added $\mathrm{Ag}$ ions and $\mathrm{Au}$ ions to the PtAu nanoframe solution at the same time and then added ascorbic acid. In this step, an Ag UPD layer was formed on the entire surface of PtAu nanoframes, and then $\mathrm{Au}$ ions were subsequently deposited on the whole surface of PtAu nanoframe ${ }^{20,21}$. No galvanic replacement reaction was observed. We believe that when the thick layer of Ag exists, the galvanic replacement is favorable with $\mathrm{AuCl}_{4}^{+}$ions added. However, the very thin layer of $\mathrm{Ag}$ behaves differently as compared to the thinker Ag layer under the same experimental conditions. The resulting $\mathrm{Au}$ double nanoframes (Fig. 2d, h, l, and p) exhibited thicker grown rims compared to Pt double frames (Fig. 2c, g, k, o and Supplementary Fig. 8). The successful concentric growth of Au was clearly evident in the corresponding UV-vis-NIR spectra, where the longwavelength band was gradually hypochromically shifted and became more intense as the $\mathrm{Au}$ coating around the core Pt rims proceeded (Supplementary Figs. 9 and 10). When the concentric growth mode was applied to single nanoframes, the EDS line mapping showed Pt rims in the cores of rims (Fig. $3 f-i$ ), different from the case of eccentric growth mode. Interestingly, X-ray diffraction (XRD) patterns of Pt@Au single frames obtained by means of eccentric growth mode indicated only two different crystalline peaks (i.e., $\{111\}$ and $\{222\}$ ), whereas Pt@Au rings synthesized by means of concentric growth exhibited several crystalline peaks (i.e., $\{111\},\{200\},\{220\}$, and $\{222\}$;
Supplementary Fig. 11). The presence of a trace Ag layer facilitated faster deposition of $\mathrm{Au}$ on both $\mathrm{Pt}$ and $\mathrm{Au}$ domains, leading to the appearance of various crystalline facets.

Electric-field simulation and assembly of $2 \mathrm{D}$ nanoframes. The Pt@Au double nanoframes were expected to show their unique near-field electromagnetic field coupling depending on the shape and the distance between the inner and outer rims (Fig. $4 \mathrm{a}-\mathrm{p}$ ). We carried out theoretical calculations using a conventional finite-difference time-domain method, finding that the electromagnetic field became coupled and enhanced as the rim-to-rim distance narrowed, regardless of shape. Interestingly, the field of rings with double frames was exclusively confined in the internal regions within nanoframes, whereas the triangular and hexagonal shapes exhibited tip-enhanced electromagnetic fields in addition to the internally confined field. In contrast, the tripod showed an internally confined field that gradually disappeared as the rim-torim gap narrowed. It is obvious that the capability of containing high volumes of molecules in the inner space between two rims and their unique electromagnetic fields inside and outside the cavity enable the design of highly effective surface-enhanced molecular sensing platforms ${ }^{22-26}$.

The resulting single or double nanoframes were exceptionally homogeneous in both size and shape, and therefore are expected to self-assemble into $3 \mathrm{D}$-ordered superstructures ${ }^{27-30}$ during simple drop-casting and evaporation. Typically, the single and double nanoframes of ring shape exhibited phenomenal longrange order (of millimeter scale) and compactness with hexagonal pattern arrangement (exemplified by Fig. 4q, r, respectively). Careful investigation of their packing order revealed that the nanoframes were stacked in a rim-to-rim fashion, paving circular channels toward the bottom. It is well known that ordered pores 
a
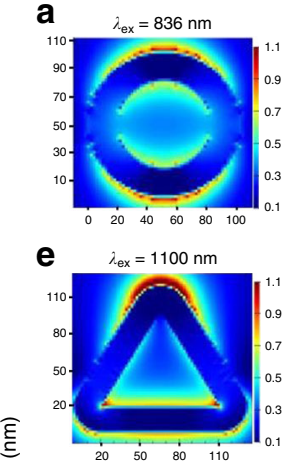

i

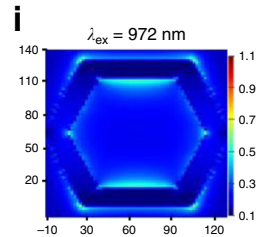

m

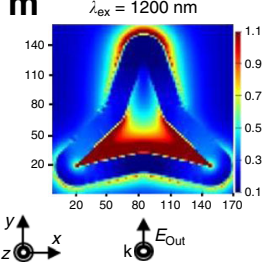

b

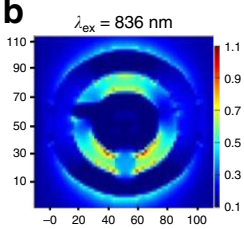

f $\lambda_{\mathrm{ex}}=1100 \mathrm{~nm}$
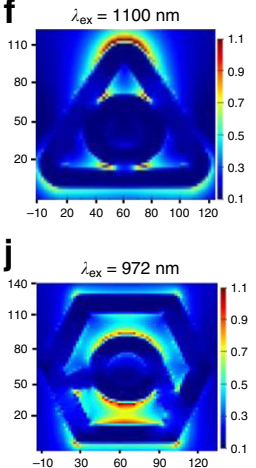

n

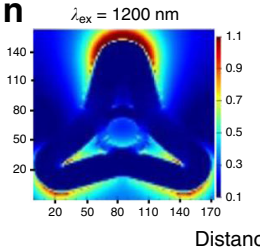

C
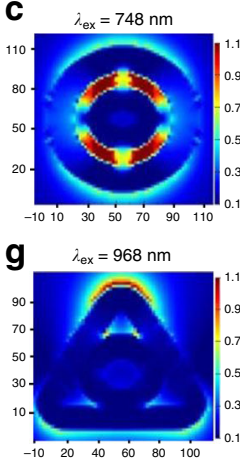

$\mathbf{K}$

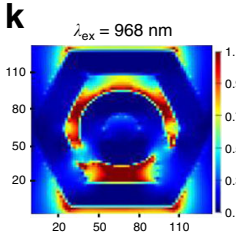

$\lambda_{\text {ex }}=980 \mathrm{~nm}$

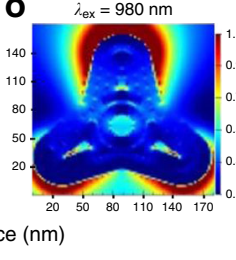

d

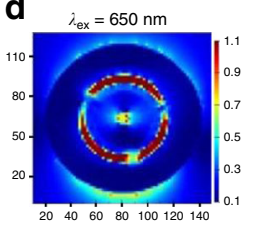

h
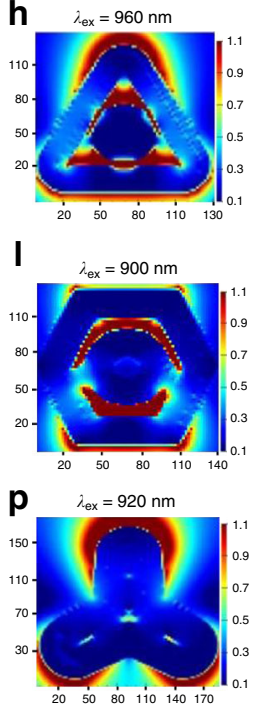

q

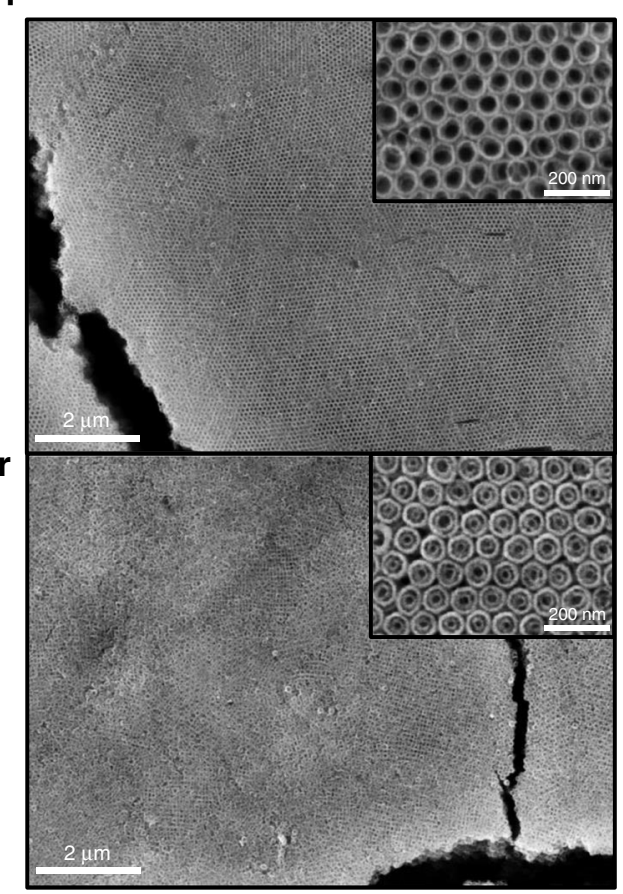

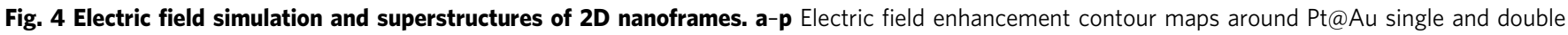

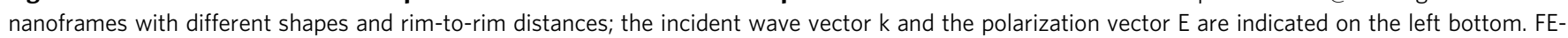
SEM images of the superstructure of (q) 2D PtAu single nanorings and ( $\mathbf{r}$ ) 2D PtAu double nanorings. Insets show zoomed-in images of the superstructures of 2D PtAu single nanorings and 2D PtAu double nanorings.

have many useful applications such as in sorbents, supercapacitors, filters, and catalysts ${ }^{31}$.

In conclusion, we demonstrated a rational and stepwise synthetic strategy for complex, highly intricate nanoplate architectures, leading to rings, triangles, hexagons, and tripods with tailorable single or double frames, resembling total synthesis of complex organic molecules. The resulting $2 \mathrm{D}$ double nanoframes with large surface area and tunability in rim-to-rim distances are expected to open various applications in catalysis, nanophotonics, and biosensing ${ }^{32-35}$.

\section{Methods}

Synthesis of 2D Au triangular nanoplates. Au triangular nanoplates were prepared from $5 \mathrm{~nm}$ sphere seeds by means of a three-step seed-mediated method with iodide ions, as reported previously ${ }^{2} .500 \mu \mathrm{L}$ of $20 \mathrm{mM}$ aqueous $\mathrm{HAuCl}_{4} \cdot 3 \mathrm{H}_{2} \mathrm{O}$ solution, $1 \mathrm{~mL}$ of a $10 \mathrm{mM}$ aqueous solution of sodium citrate, and $1 \mathrm{~mL}$ of $100 \mathrm{mM}$ aqueous $\mathrm{NaBH}_{4}$ (ice-cold) solution were added to $36.5 \mathrm{~mL}$ of deionized water under vigorous stirring. Three labeled flasks were used to prepare the triangular nanoplates. A mixture of $108 \mathrm{~mL}$ of $0.05 \mathrm{M}$ aqueous CTAB solution and $54 \mu \mathrm{L}$ of $0.1 \mathrm{M}$ aqueous $\mathrm{NaI}$ solution was divided into three containers labeled 1, 2, and $3.9 \mathrm{~mL}$ of the mixture were added each to containers 1 and 2 , and the remaining $90 \mathrm{~mL}$ was added to container 3. A mixture of $125 \mu \mathrm{L}$ of $20 \mathrm{mM}$ aqueous $\mathrm{HAuCl}_{4} \cdot 3 \mathrm{H}_{2} \mathrm{O}$ solution, $50 \mu \mathrm{L}$ of $100 \mathrm{mM} \mathrm{NaOH}$, and $50 \mu \mathrm{L}$ of $100 \mathrm{mM}$ ascorbic acid was then added each to containers 1 and 2 . A mixture of $1.25 \mathrm{~mL}$ of $20 \mathrm{mM}$ $\mathrm{HAuCl}_{4} \cdot 3 \mathrm{H}_{2} \mathrm{O}, 0.5 \mathrm{~mL}$ of $100 \mathrm{mM} \mathrm{NaOH}$, and $0.5 \mathrm{~mL}$ of $100 \mathrm{mM}$ ascorbic acid was added to container 3 . A total of $1 \mathrm{~mL}$ of the seed solution was added to container 1 under mild shaking, followed by the addition of $1 \mathrm{~mL}$ of the container 1 solution into container 2. After gentle shaking, all solution in container 2 was added to container 3 .

Synthesis of 2D Au circular nanoplates. Au circular nanodisks were prepared from triangular nanoplates by means of a comproportionation reaction. One and a half milliliters of a mixture of $10 \mathrm{~mL}$ of $0.1 \mathrm{M}$ CTAB solution and $250 \mu \mathrm{L}$ of $20 \mathrm{mM}$ aqueous $\mathrm{HAuCl}_{4} \cdot 3 \mathrm{H}_{2} \mathrm{O}$ solution was added to $10 \mathrm{~mL}$ of an aqueous solution of triangular nanoplates. The triangular nanoplates were etched via gold ions in an isothermal oven at $30^{\circ} \mathrm{C}$. After $1 \mathrm{~h}$, the triangular nanoplates transformed to gold nanodisks. Residual gold ions were then removed by means of centrifugation $(6511 \times g$ for $20 \mathrm{~min})$.

Synthesis of 2D Au hexagonal nanoplates. Au hexagonal nanoplates were prepared from circular nanodisks by reducing $\mathrm{HAuCl}_{4}$ solution with ascorbic acid, as reported previously ${ }^{36}$. Au hexagonal nanoplates were obtained by mixing $1 \mathrm{~mL}$ of $0.1 \mathrm{M}$ aqueous CTAB solution, $80 \mu \mathrm{L}$ of $20 \mathrm{mM} \mathrm{HAuCl}_{4}, 80 \mu \mathrm{L}$ of $100 \mathrm{mM}$ ascorbic acid and $6 \mathrm{~mL}$ of an aqueous solution of circular nanodisks. The major plasmon peak intensity of the aqueous solution of circular nanodisks was adjusted to 1.2 with water prior to mixing. The mixture was reacted by holding it in an isothermal oven at $30^{\circ} \mathrm{C}$ for $10 \mathrm{~h}$.

Synthesis of 2D Au tripod nanoplates. Au tripod nanoplates were prepared from triangular nanoplates by means of site-selective regrowth with silver ions and etching of edge sites. $4 \mathrm{~mL}$ of an aqueous solution of triangular nanoplate, $20 \mathrm{~mL}$ of $0.05 \mathrm{M}$ aqueous $\mathrm{CTAB}$ solution, $10 \mu \mathrm{L}$ of $0.1 \mathrm{M}$ aqueous $\mathrm{NaI}$ solution, $35 \mu \mathrm{L}$ of $2 \mathrm{mM}$ aqueous $\mathrm{AgNO}_{3}$, and $480 \mu \mathrm{L}$ of $100 \mathrm{mM}$ ascorbic acid were added to a $20 \mathrm{~mL}$ vial. The mixture was held in an isothermal oven at $70{ }^{\circ} \mathrm{C}$ for $1.5 \mathrm{~h}$ to deposit silver atoms onto the surfaces of the triangular nanoplates. After washing, the aqueous solution of Au@Ag triangular nanoplate was diluted to $15 \mathrm{~mL}$ by adding distilled water. Then, $2.5 \mathrm{~mL}$ of the aqueous solution of diluted Au@Ag triangular nanoplate, $5 \mathrm{~mL}$ of $0.05 \mathrm{M}$ aqueous CTAB solution, $2.5 \mu \mathrm{L}$ of $0.1 \mathrm{M}$ aqueous $\mathrm{NaI}$ solution, $200 \mu \mathrm{L}$ of $2 \mathrm{mM}$ aqueous $\mathrm{HAuCl}_{4}$ solution and $100 \mu \mathrm{L}$ of $100 \mathrm{mM}$ ascorbic acid were added to a $20 \mathrm{~mL}$ vial. The resulting mixture was held in an isothermal oven at $70^{\circ} \mathrm{C}$ for $3 \mathrm{~h}$. The selective reduction step was monitored by means of extinction spectrum measurements. Residual ions were then removed by means of centrifugation $(6511 \times g$ for $20 \mathrm{~min})$.

Synthesis of 2D PtAu single nanoframes. The synthetic pathway for siteselective growth of Pt on Au nanoplates (Au@Pt nanoplates) followed our previously reported experimental procedure ${ }^{15}$. In the presence of iodide ions $(50 \mu \mathrm{M})$, $20 \mathrm{~mL}$ of $0.05 \mathrm{M} \mathrm{CTAB}, 4 \mathrm{~mL}$ of redispersed Au nanoplates with different shapes (optical density of disk, prism, hexagonal, and tripod is $1.2,1,1$, and 1 , respectively), $20 \mu \mathrm{L}(1.6 \mu \mathrm{M})$ of $2 \mathrm{mM}$ aqueous $\mathrm{AgNO}_{3}$ solution, and $480 \mu \mathrm{L}(2 \mathrm{mM})$ of $0.1 \mathrm{M}$ aqueous ascorbic acid solution were added to a vial. The mixture was kept at $70^{\circ} \mathrm{C}$. After $1 \mathrm{~h}, 480 \mu \mathrm{L}(2 \mathrm{mM})$ of $0.1 \mathrm{M} \mathrm{HCl}$ and $100 \mu \mathrm{L}(8.3 \mu \mathrm{M})$ (disks), $175 \mu \mathrm{L}$ (14.5 $\mu \mathrm{M})$ (triangles), $150 \mu \mathrm{L}(12.5 \mu \mathrm{M})$ (hexagons), and $250 \mu \mathrm{L}(20.8 \mu \mathrm{M})$ (tripods) of $2 \mathrm{mM}$ aqueous $\mathrm{H}_{2} \mathrm{PtCl}_{6}$ solution were added to the mixture with gentle shaking. 
The mixture was kept at $70{ }^{\circ} \mathrm{C}$ for $4 \mathrm{~h}$. After this reaction, the sample was centrifuged and the supernatant was removed and redispersed in a mixture of $5 \mathrm{~mL}$ of $0.05 \mathrm{M}$ CTAB aqueous solution and $2.5 \mu \mathrm{L}$ of $0.1 \mathrm{M}$ aqueous $\mathrm{NaI}$ solution. Next, $500 \mu \mathrm{L}$ of $2 \mathrm{mM}$ aqueous $\mathrm{HAuCl}_{4}$ was added to etch the Au@Pt nanoplates. This etching process was carried out for $1 \mathrm{~h}$ in a $50{ }^{\circ} \mathrm{C}$ oven and was followed by centrifugal washing $(6511 \times \mathrm{g}$ for $20 \mathrm{~min})$.

Eccentric growth of 2D Pt@Au single nanoframes. Au frame nanoparticles with eccentric growth mode were prepared by reducing gold ions. A solution of Pt frame nanoparticles was stabilized by adding a mixture of $10 \mathrm{~mL}$ of $0.05 \mathrm{M}$ CTAB aqueous solution and $5 \mu \mathrm{L}$ of $0.1 \mathrm{M}$ aqueous $\mathrm{NaI}$ solution. Aqueous $2 \mathrm{mM} \mathrm{HAuCl}$ solution was added to $\mathrm{Pt}$ frame nanoparticle solution in the following volumes: $200 \mu \mathrm{L}(40 \mu \mathrm{M}), 400 \mu \mathrm{L}(80 \mu \mathrm{M})$, and $600 \mu \mathrm{L}(60 \mu \mathrm{M})$ for disks. Then, $100 \mathrm{mM}$ ascorbic acid was added to the resulting mixtures in a 1:10 volume ratio. The resulting reduction reaction was monitored by means of extinction spectrum measurements. Residual ions were then removed by means of centrifugation $(6511 \times g$ for $20 \mathrm{~min})$

Synthesis of 2D PtAu double nanoframes. In the presence of iodide ions $(50 \mu \mathrm{M}), 20 \mathrm{~mL}$ of $0.05 \mathrm{M} \mathrm{CTAB}, 4 \mathrm{~mL}$ of redispersed inwardly grown $\mathrm{Au}$ frame nanoparticles of eccentric growth mode, $16 \mu \mathrm{L}(1.3 \mu \mathrm{M})$ (disks), $15 \mu \mathrm{L}(1.3 \mu \mathrm{M})$ (triangles), $16 \mu \mathrm{L}(1.25 \mu \mathrm{M})$ (hexagons), and $12 \mu \mathrm{L}(1 \mu \mathrm{M})$ (tripods) of $2 \mathrm{mM}$ aqueous $\mathrm{AgNO}_{3}$ solution, and $480 \mu \mathrm{L}(2 \mathrm{mM})$ of $0.1 \mathrm{M}$ aqueous ascorbic acid solution were added to vials. Each resulting mixture was held at $70^{\circ} \mathrm{C}$. After $1 \mathrm{~h}$, $480 \mu \mathrm{L}(2 \mathrm{mM})$ of $0.1 \mathrm{M} \mathrm{HCl}$ and $96 \mu \mathrm{L}(8 \mu \mathrm{M})$ (disks), $120 \mu \mathrm{L}(10 \mu \mathrm{M})$ (triangles), $80 \mu \mathrm{L}(6.7 \mu \mathrm{M})$ (hexagons), or $120 \mu \mathrm{L}(10 \mu \mathrm{M})$ (tripods) of $2 \mathrm{mM}$ aqueous $\mathrm{H}_{2} \mathrm{PtCl}_{6}$ solution were added to the respective mixtures with gentle shaking. Each sample was reacted at $70^{\circ} \mathrm{C}$ for $4 \mathrm{~h}$ and then it was centrifuged two times. The supernatant holding the resulting Au@Pt nanoplates was removed and redispersed in a mixture of $4 \mathrm{~mL}$ of $0.05 \mathrm{M}$ CTAB aqueous solution and $2.5 \mu \mathrm{L}$ of $0.1 \mathrm{M}$ aqueous $\mathrm{NaI}$ solution. Next, $250 \mu \mathrm{L}(125 \mu \mathrm{M})$ of $2 \mathrm{mM}$ aqueous $\mathrm{HAuCl}_{4}$ was added to etch the nanoplates. This etching process was carried out for $1 \mathrm{~h}$ in a $50^{\circ} \mathrm{C}$ oven and was followed by centrifugal washing $(6511 \times g$ for $20 \mathrm{~min})$.

Growth of 2D Pt@Au double nanoframes. Pt@Au double nanoframes were prepared by means of Au regrowth on Pt frame nanoparticles with a small amount of silver ions. A solution of the synthesized PtAu frame nanoparticles was stabilized by adding a mixture of $4 \mathrm{~mL}$ of $0.05 \mathrm{M} \mathrm{CTAB}$ aqueous solution and $2.5 \mu \mathrm{L}$ of $0.1 \mathrm{M}$ aqueous $\mathrm{NaI}$ solution. Before the Au reduction reaction, $1 \mu \mathrm{L}(0.5 \mu \mathrm{M})$ of $2 \mathrm{mM}$ aqueous $\mathrm{AgNO}_{3}$ was added. Aqueous $2 \mathrm{mM} \mathrm{HAuCl}$ solution was added to $\mathrm{Pt}$ frame nanoparticle solution in the following volumes: $50 \mu \mathrm{L}(25 \mu \mathrm{M}), 100 \mu \mathrm{L}$ $(50 \mu \mathrm{M})$, and $150 \mu \mathrm{L}(75 \mu \mathrm{M})$ for disks and prisms, $50 \mu \mathrm{L}(25 \mu \mathrm{M}), 100 \mu \mathrm{L}(50 \mu \mathrm{M})$, and $200 \mu \mathrm{L}(100 \mu \mathrm{M})$ for hexagons, and $60 \mu \mathrm{L}(30 \mu \mathrm{M}), 180 \mu \mathrm{L}(90 \mu \mathrm{M})$, and $240 \mu \mathrm{L}$ $(120 \mu \mathrm{M})$ for tripods. Then, $100 \mathrm{mM}$ ascorbic acid was added to the resulting mixtures in a 1:10 volume ratio. The resulting reduction reaction was monitored by means of extinction spectrum measurements. Residual ions were then removed by means of centrifugation $(6511 \times g$ for $20 \mathrm{~min})$.

Electric field simulations. Simulations of the electric field near the nanoframe were performed by means of a conventional FDTD method (FDTD Solutions, LumericalTM). Dimensions of nanoframes were indicated in Supplementary Fig. 10 for detail. The simulation volume of $140 \mathrm{~nm} \times 140 \mathrm{~nm} \times 1.3 \mu \mathrm{m}$ was used to completely cover the nanoframe structure and the light source. A vertically polarized light source (polarized along the $y$ direction in Fig. 4) of plane wave form for each simulated wavelength was positioned $300 \mathrm{~nm}$ away from the nanoframes, and a monitor of dimensions $123 \mathrm{~nm} \times 123 \mathrm{~nm}$ was positioned at the center of the nanoframes. To ensure precise simulation, the minimum mesh size was set to $0.1 \mathrm{~nm}$.

Characterization. Field emission scanning electron microscopy (FESEM) images were obtained using JSM-7100F and JSM-7800F instruments (JEOL). JEM-2100F and JEM-ARM $200 \mathrm{~F}$ instruments (JEOL) were used to acquire transmission electron microscopy (TEM) images. UV-vis-NIR absorption spectra were acquired using a spectrophotometer (Shimadzu UV-3600). 3D tomography images were obtained using Talos F200X. A NX10 instrument (Park systems) was used to acquire AFM images and height profiles. X-ray diffraction (XRD) patterns of the nanostructures were characterized using a Rigaku Ultima IV. The electrochemical measurements were performed using an Auto Lab AUT12.

\section{Data availability}

The authors declare that all data are available from the corresponding authors on reasonable request

Received: 19 July 2019; Accepted: 22 November 2019;

Published online: 19 December 2019

\section{References}

1. Burrows, N. D. et al. Anisotropic nanoparticles and anisotropic surface chemistry. J. Phys. Chem. Lett. 7, 632-641 (2016).

2. Scarabelli, L., Coronado-Puchau, M., Giner-Casares, J. J., Langer, J. \& LizMarzán, L. M. Monodisperse gold nanotriangles: size control, large-scale selfassembly, and performance in surface-enhanced Raman scattering. ACS Nano 8, 5833-5842 (2014).

3. Jin, R. et al. Photoinduced conversion of silver nanospheres to nanoprisms. Science 294, 1901-1903 (2001).

4. Straney, P. J., Andolina, C. M. \& Millstone, J. E. Seedless initiation as an efficient, sustainable route to anisotropic gold nanoparticles. Langmuir 29, 4396-4403 (2013).

5. Huo, D. et al. Seed-mediated growth of Au nanospheres into hexagonal stars and the emergence of a hexagonal close-packed phase. Nano Lett. 19, 3115-3121 (2019).

6. González, E., Arbiol, J. \& Puntes, V. F. Carving at the nanoscale: sequential galvanic exchange and kirkendall growth at room temperature. Science 334, 1377-1380 (2011).

7. Chen, P. et al. Polyelemental nanoparticle libraries. Science 352, 1565-1569 (2016).

8. Yu, Y., Zhang, Q., Xie, J. \& Lee, J. Y. Engineering the architectural diversity of heterogeneous metallic nanocrystals. Nat. Commun. 4, 1454 (2013).

9. Buck, M. R., Bondi, J. F. \& Schaak, R. E. A total-synthesis framework for the construction of high-order colloidal hybrid nanoparticles. Nat. Chem. 4, 37-44 (2012).

10. Fenton, J. L., Steimle, B. C. \& Schaak, R. E. Tunable intraparticle frameworks for creating complex heterostructured nanoparticle libraries. Science $\mathbf{3 6 0}$, 513-517 (2018).

11. Li, X. F. \& Schaak, R. E. Reactive AgAuS and Ag3AuS2 synthons enable the sequential transformation of spherical nanocrystals into asymmetric multicomponent hybrid nanoparticles. Chem. Mater. 29, 4153-4160 (2017).

12. Hong, S., Shuford, K. L. \& Park, S. Shape transformation of gold nanoplates and their surface plasmon characterization: triangular to hexagonal nanoplates. Chem. Mater. 23, 2011-2013 (2011).

13. Chen, S. H., Wang, Z. L., Ballato, J., Foulger, S. H. \& Carroll, D. L. Monopod, bipod, tripod, and tetrapod gold nanocrystals. J. Am. Chem. Soc. 125, 16186-16187 (2003)

14. Hao, E., Bailey, R. C., Schatz, G. C., Hupp, J. T. \& Li, S. Synthesis and optical properties of branched gold nanocrystals. Nano Lett. 4, 327-330 (2004).

15. Jang, H. J. et al. Shape-controlled synthesis of Pt nanoframes. J. Mater. Chem. 22, 19792-19797 (2012)

16. Hajfathalian, M. et al. A Wulff in a cage: the confinement of substrate-based structures in plasmonic nanoshells, nanocages, and nanoframes using galvanic replacement. ACS Nano 10, 6354-6362 (2016).

17. Park, J. et al. Iridium-based multimetallic nanoframe@nanoframe structure: an efficient and robust electrocatalyst toward oxygen evolution reaction. ACS Nano 11, 5500-5509 (2017).

18. Kwon, H. et al. Dendrite-embedded platinum-nickel multiframes as highly active and durable electrocatalyst toward the oxygen reduction reaction. Nano Lett. 18, 2930-2936 (2018).

19. Han, F. et al. On demand synthesis of hollow fullerene nanostructures. Nat. Commun. 10, 1548 (2019)

20. Personick, M. L., Langille, M. R., Zhang, J. \& Mirkin, C. A. Shape control of gold nanoparticles by silver underpotential deposition. Nano Lett. 11, 3394-3398 (2011)

21. Grzelczak, M. Silver ions direct twin-plane formation during the overgrowth of single-crystal gold nanoparticles. ACS Omega 1, 177-181 (2016).

22. Lim, D. K. Highly uniform and reproducible surface-enhanced Raman scattering from DNA-tailorable nanoparticles with 1-nm interior gap. Nat. Nanotechnol. 6, 452-460 (2011).

23. Jiang, T. Construction of long narrow gaps in Ag nanoplates. J. Am. Chem. Soc. 140, 15560-15563 (2018)

24. Lerch, S. \& Reinhard, B. M. Effect of interstitial palladium on plasmon-driven charge transfer in nanoparticle dimers. Nat. Commun. 9, 1608 (2018).

25. Nam, J. M., Oh, J. O., Lee, H. \& Suh, Y. D. Plasmonic nanogap-enhanced Raman scattering with nanoparticles. ACC Chem. Res. 49, 2746-2755 (2016)

26. Shen, W. et al. Reliable quantitative SERS analysis facilitated by core-shell nanoparticles with embedded internal standards. Angew. Chem. Int. Ed. 54, 7308-7312 (2015).

27. Kang, Y. J. et al. Design of Pt-Pd binary superlattices exploiting shape effects and synergistic effects for oxygen reduction reactions. J. Am. Chem. Soc. 135, 42-45 (2013)

28. Wang, T. Self-assembled colloidal superparticles from nanorods. Science 338, 358-363 (2012).

29. Xia, Y. et al. Self-assembly of self-limiting monodisperse supraparticles from polydisperse nanoparticles. Nat. Nanotechnol. 6, 580-587 (2011) 
30. Bourgeois, M. R., Liu, A. T., Ross, M. B., Berlin, J. M. \& Schatz, G. C. SelfAssembled plasmonic metamolecules exhibiting tunable magnetic response at optical frequencies. J. Phys. Chem. C. 121, 15915-15921 (2017).

31. Yoo, S. et al. 3D PtAu nanoframe superstructure as a high-performance carbon-free electrocatalyst. Nanoscale 11, 2840-2847 (2019).

32. Niu, Z. Q. et al. Anisotropic phase segregation and migration of Pt in nanocrystals en route to nanoframe catalysts. Nat. Mater. 15, 1188 (2016).

33. Sonnefraud, Y. et al. Experimental realization of subradiant, superradiant, and Fano resonances in ring/disk plasmonic nanocavities. ACS Nano 4, 1664-1670 (2010).

34. Mukherjee, S. et al. Fanoshells: nanoparticles with built-in Fano resonances. Nano Lett. 10, 2694-2701 (2010).

35. Near, R., Tabor, C., Duan, J., Pachter, R. \& El-Sayed, M. Pronounced effects of anisotropy on plasmonic properties of nanorings Fabricated by electron beam lithography. Nano Lett. 12, 2158-2164 (2012).

36. Qin, F. et al. Thickness control produces gold nanoplates with their plasmon in the visible and near-infrared regions. Adv. Optical Mater. 4, 76-85 (2016).

\section{Acknowledgements}

This research was supported by the Basic Science Research Program through the National Research Foundation of Korea (NRF) funded by the Ministry of Science, ICT and Future Planning (NRF-2017R1A2B3002796), the National Research Foundation of Korea (NRF) grant funded by the Korea government (MSIP) (NRF-2017R1A5A1015365) and Agency for Defense Development through Chemical and Biological Detection Research Center (UD170024ID).

\section{Author contributions}

S.Y. and J.K. performed the experiments and data analysis. S.P. and S.Y. wrote the paper. S.C. and D.J.P. simulated FDTD method for electromagnetic field enhancement. S.P. designed and supervised the project. All authors discussed the results and commented on the paper.

\section{Competing interests}

The authors declare no competing interests.

\section{Additional information}

Supplementary information is available for this paper at https://doi.org/10.1038/s41467019-13738-6.

Correspondence and requests for materials should be addressed to S.P.

Peer review information Nature Communications thanks the anonymous reviewers for their contribution to the peer review of this work.

Reprints and permission information is available at http://www.nature.com/reprints

Publisher's note Springer Nature remains neutral with regard to jurisdictional claims in published maps and institutional affiliations.

(c) (i) Open Access This article is licensed under a Creative Commons Attribution 4.0 International License, which permits use, sharing, adaptation, distribution and reproduction in any medium or format, as long as you give appropriate credit to the original author(s) and the source, provide a link to the Creative Commons license, and indicate if changes were made. The images or other third party material in this article are included in the article's Creative Commons license, unless indicated otherwise in a credit line to the material. If material is not included in the article's Creative Commons license and your intended use is not permitted by statutory regulation or exceeds the permitted use, you will need to obtain permission directly from the copyright holder. To view a copy of this license, visit http://creativecommons.org/ licenses/by/4.0/.

(C) The Author(s) 2019 